\title{
Review of: "Intensification of El Niño-induced atmospheric anomalies under greenhouse warming"
}

\author{
kwang-yul kim \\ 1 Seoul National University
}

Potential competing interests: The author(s) declared that no potential competing interests exist.

Review of: "Intensification of El Niño-induced atmospheric anomalies under greenhouse warming"

Kwang-Yul Kim ${ }^{1}$

${ }^{1}$ Seoul National University

The recent Nature Geoscience paper "Intensification of El Niño-Induced atmospheric anomalies under greenhouse warming" is an interesting article, suggesting that greenhouse warming can significantly intensify the atmospheric response to El Niño. The main mechanism of this intensification is increased specific humidity owing to atmospheric warming leading to increased saturation specific humidity (Clausius-Clapeyron relationship). This, in fact, appears to be the major vertical feedback mechanism explaining a rapid warming of the Arctic atmosphere (Kim and Kim 2019). The veracity of the conclusion drawn in the paper based on the CMIP6 (Climate Model Intercomparison Project Version 6) datasets, however, seems to require more careful scrutiny against observation or reanalysis data.

Monthly extended reconstructed sea surface temperatures (ERSST; Huang et al. 2017) from 1979 to 2020 are used to investigate whether the same conclusion can be drawn in the ERA5 reanalysis data (Hersbach et al. 2020). Figure 1a shows SST change from the PRE (1979-1999) to the POST (2000-2020) periods. As can be seen, SST change is fairly substantial, leading up to $\sim 1 \mathrm{~K}$ sea surface warming between the two intervals (20 years). During the same period, the mean rate of $\mathrm{CO}_{2}$ increase is $\sim 0.5 \% \mathrm{yr}^{-1}$, leading to 78 PPM increase from 1979 to 2020, based on the time series at Mauna Loa (https://gml.noaa.gov/ccgg/trends/ data.html). Thus, the rate of warming in the reanalysis data seems somewhat lower per mean rate of $\mathrm{CO}_{2}$ increase than that in the CMIP6 MME (see Fig. 1a in Hu et al. 2021), although temperature response is not necessarily linearly proportional to the rate of $\mathrm{CO}_{2}$ increase. Nonetheless, it is clear that SST has increased due to the increasing concentration of $\mathrm{CO}_{2}$. Based on the first CSEOF (global warming) mode (see supplementary information), it is estimated that the mean rate of warming is $\sim 0.008 \mathrm{~K} \mathrm{yr}^{-1}$ in the latitude band of $10^{\circ} \mathrm{S}-10^{\circ} \mathrm{N}$ (see also Figs. S1-S3). After removing the global warming mode, SST change is essentially zero in the whole domain as should be expected (Fig. 1b). Figure 2 shows the NINO3.4 $\left(170^{\circ}-120^{\circ} \mathrm{W}, 5^{\circ} \mathrm{S}-5^{\circ} \mathrm{N}\right)$ time series derived from the ERSST data after the trend is removed. This time series is similar to the NINO3.4 time series published elsewhere (e.g., 
NOAA/NCEI) except for the trend. Regression analysis is conducted in the form

\section{$\underline{Y(r, t)=a(r) X(t)+b(r)}$}

, where

\section{$X(t)$}

is the NINO3.4 index time series and

\section{$Y(r, t)$}

is a variable for which the regression pattern is desired. The parameter

\section{$a(r)$}

is determined via

$$
a(r)=\sum_{t=1}^{N} X(t) Y(r, t) / \sum_{t=1}^{N} X(t) X(t)
$$

where

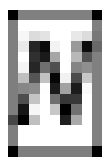

is the number of time points. Regression analysis is conducted by dividing the record into two parts: PRE (1979-1999) and POST (2000-2020). It is noted that linear trend is removed from

\section{$Y(r, t)$}

prior to the regression analysis, although similar results are obtained when linear trend is not removed. Figures 3-7 show the regressed patterns of the variables analyzed. All the regressed patterns are for winter (DJF) and depict the familiar El Niño conditions. Vertical velocity is shown to increase in the central and eastern equatorial Pacific due to surface warming (Fig. 3a and b), and a descending motion is shown in the far-western Pacific (Fig. 3d and e). Due to increased evaporation (and also air temperature potentially), specific humidity in the atmospheric column increases in the equatorial Pacific (Fig. 4a and b). At the same time, a significant increase in precipitation is also seen (Fig. 4d and e). The 200-hPa geopotential and temperature patterns exhibit the familiar "dumb-bell" shapes (Fig. 5). Figures 6 and 7 further show the effect of El Niño in the equatorial $\left(5^{\circ} \mathrm{S}-5^{\circ} \mathrm{N}\right)$ troposphere with warming, moistening, and weakened Walker circulation.

Despite the global warming, however, there is no sign of intensification of El Niño signal in the atmosphere according to the ERA5 reanalysis data. All the variables examined display no substantial change from the PRE to the POST periods particularly in the eastern equatorial Pacific (see panels $c$ and $f$ of Figs. 3-7). Specifically, there is no sign of substantial tropospheric warming in association with El Niño (Fig. 6f) and subsequent increase in (saturation) specific humidity (Fig. 7c). A comparison with the ERA5 reanalysis data 
indicates that an intensification of atmospheric response to ENSO may be specific to CMIP6 models. It should be pointed out that the conclusion drawn by the authors using the CMIP6 models does not necessarily hold in the real world.

Hersbach H, et al. (2020) The ERA5 Global Reanalysis. Quar J Royal Meteorol Soc, 146:1999-2049. Hu K, Huang G, Huang P, Kosaka Y, and Xie S-P (2021) Intensification of El Nino-induced atmospheric anomalies under greenhouse warming. Nat Geosci 14:377-382.

Huang B, Thorne PW, Banzon VF, Boyer T, Chepurin G, Lawrimore JH, Menne MJ, Smith TM, Vose RS, and Zhang H-M (2017) Extended Reconstructed Sea Surface Temperature, version 5 (ERSSTv5): Upgrades, validations, and intercomparisons. J Clim 30:8179-8205.

Kim J-Y, and Kim K-Y (2019) Relative role of horizontal and vertical processes in the physical mechanism of wintertime Arctic amplification. Clim Dyn 52:6097-6107.

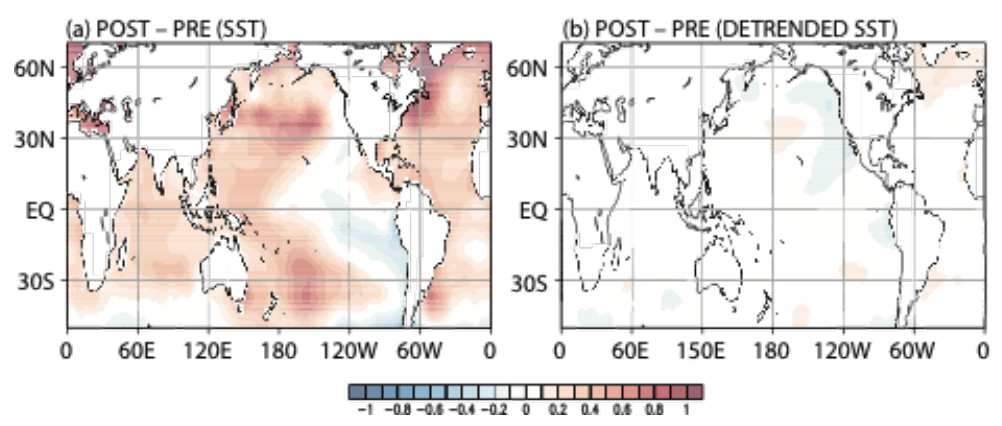

Figure 1. (a) Difference in sea surface temperature between POST and PRE. (b) Same as (a) except for the detrended sea surface temperature (without the global warming mode).

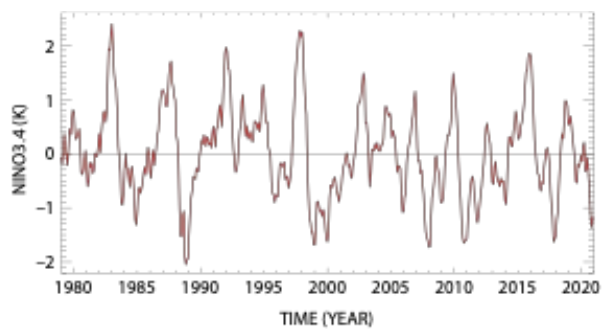

Figure 2. Nino3.4 index time series derived from the ERSST data after removing the global warming mode. 

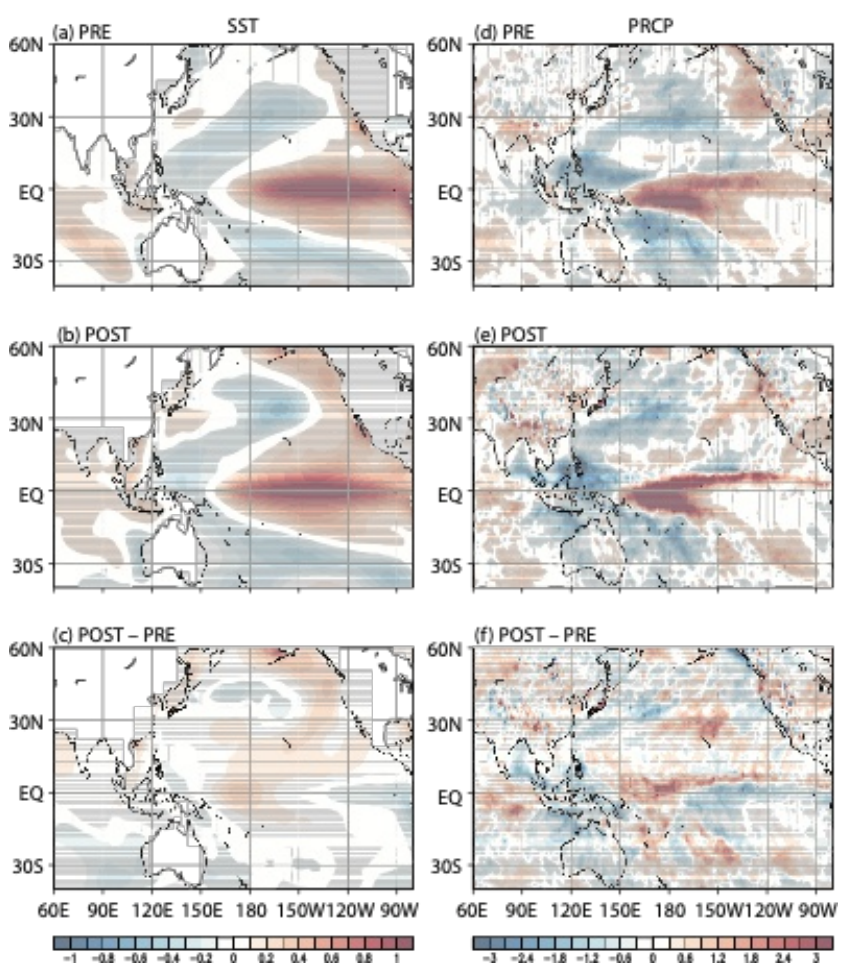

Figure 3. Regression coefficients for ( $a$ and $d$ ) the earlier period (PRE; 1979-1999), (b and e) the later period (POST; 2000-2020), and (c and f) the difference between the two (POST-PRE); (a-c) for sea surface temperature $(K)$ and $(d-f) 500-h P a$ vertical velocity $\left(-0.01 \mathrm{~Pa} \mathrm{~s}^{-1}\right)$ in winter (DJF).
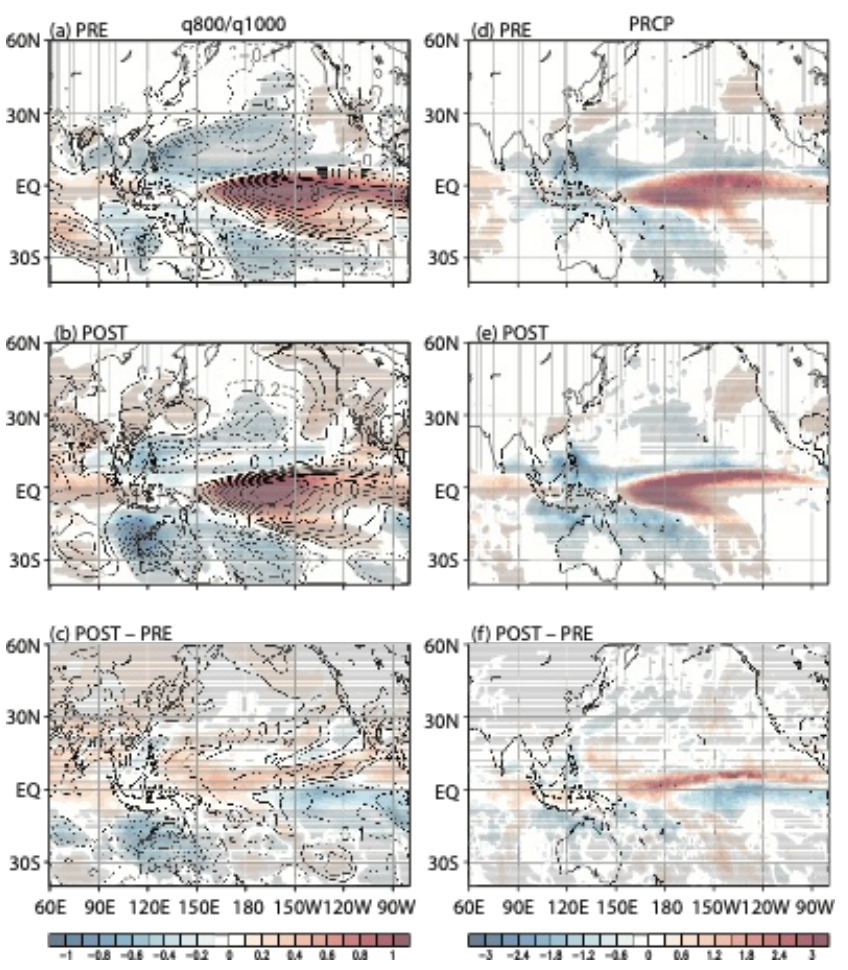

Figure 4. Regression coefficients for ( $a$ and $d$ ) the earlier period (PRE; 1979-1999), (b and e) the later 
period (POST; 2000-2020), and ( $c$ and f) the difference between the two (POST-PRE); (a-c) for 1000-and 850-hPa specific humidity $\left(\mathrm{g} \mathrm{kg}^{-1}\right)$ and (d-f) total precipitation ( $\left.\mathrm{mm} \mathrm{day}^{-1}\right)$ in winter (DJF).
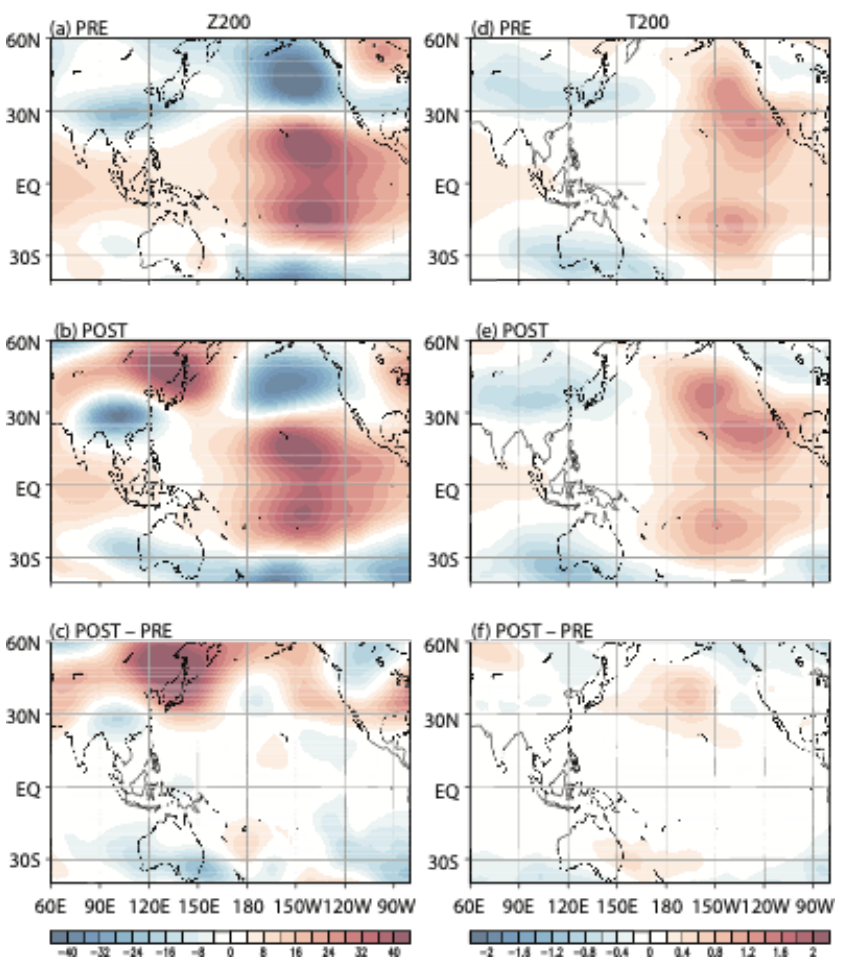

Figure 5. Regression coefficients for ( $a$ and $d$ ) the earlier period (PRE; 1979-1999), (b and e) the later period (POST; 2000-2020), and (c and f) the difference between the two (POST-PRE); (a-c) for 200-hPa geopotential height $(\mathrm{m})$ and $(\mathrm{d}-\mathrm{f})$ 200-hPa temperature (K) in winter (DJF).
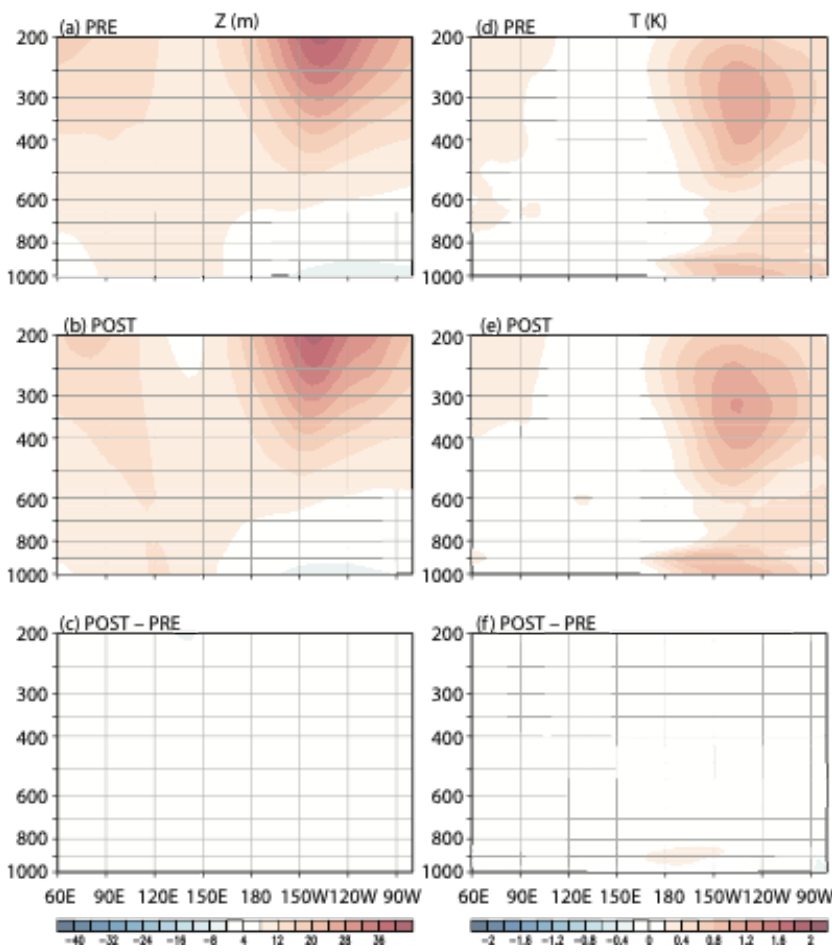
Figure 6. Regression coefficients for ( $a$ and $d$ ) the earlier period (PRE; 1979-1999), (b and e) the later period (POST; 2000-2020), and (c and f) the difference between the two (POST-PRE); (a-c) for 1000-200hPa geopotential height $(\mathrm{m})$ and $(\mathrm{d}-\mathrm{f})$ 1000-200-hPa temperature (K) in winter (DJF).
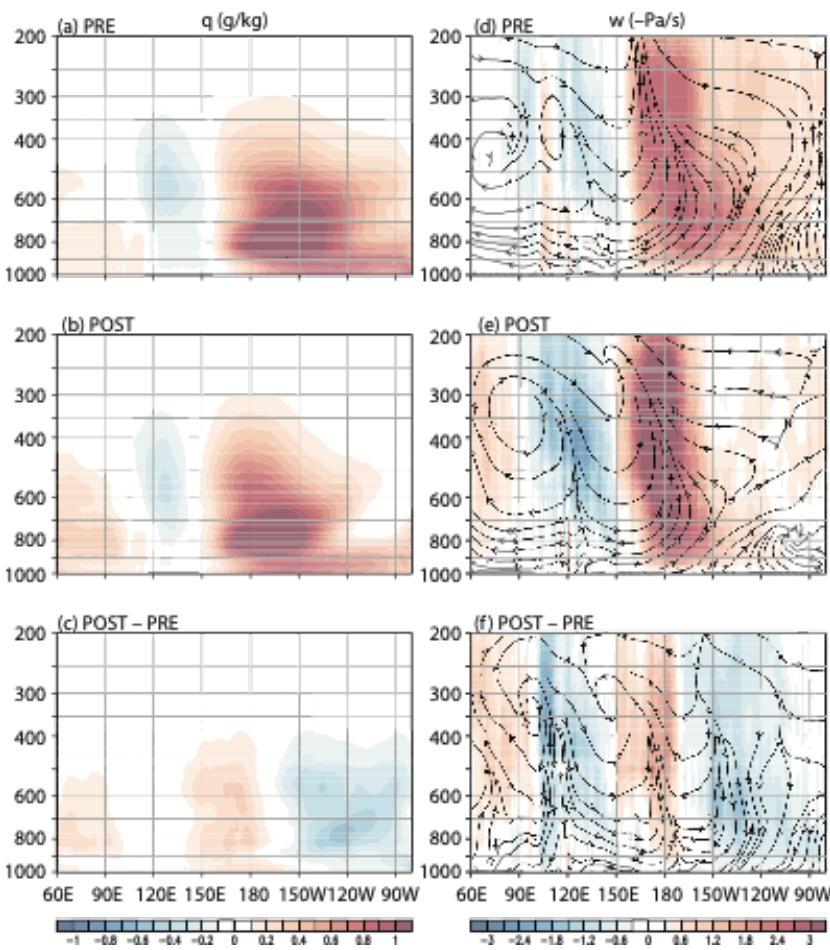

\section{Figure 7}

. Regression coefficients for ( $a$ and $d$ ) the earlier period (PRE; 1979-1999), (b and e) the later period (POST; 2000-2020), and (c and f) the difference between the two (POST-PRE); (a-c) for 1000-200-hPa specific humidity $(\mathrm{g} \mathrm{kg}$

$-1$

) and (d-f) 1000-200-hPa vertical velocity (-Pa S

$-1$

) and circulation in winter (DJF). 